\title{
COVID-19 Pandemic Causing Acute Kidney Injury and Impact on Patients With Chronic Kidney Disease and Renal Transplantation
}

\author{
Sreedhar Adapa ${ }^{\mathrm{a}, \mathrm{j}}$, Avantika Chenna ${ }^{\mathrm{b}}$, Mamtha Balla ${ }^{\mathrm{c}}$, Ganesh Prasad Merugu ${ }^{\mathrm{d}}$, \\ Narayana Murty Kodurie, Subba Rao Daggubatif ${ }^{\mathrm{f}}$, Vijay Gayam ${ }^{\mathrm{g}}$, \\ Srikanth Naramala ${ }^{\mathrm{h}}$, Venu Madhav Konala ${ }^{\mathrm{i}}$
}

\begin{abstract}
Coronavirus disease 2019 (COVID-19) caused by 2019 novel coronavirus (2019-nCoV) has caused significant mortality and has been declared as a global pandemic by the World Health Organization. The infection mainly presents as fever, cough, and breathing difficulty, and few patients develop very severe symptoms. The purpose of this review is to analyze the impact of the virus on the kidney. COVID-19 infection causes acute kidney injury (AKI) and is an independent risk factor for mortality. Angiotensin-converting enzyme 2 (ACE2) receptors, direct viral damage, and immune-mediated damage play important roles in the pathogenesis. AKI in COVID-19 infection could be from the synergistic effect of virus-induced direct cytotropic effect and cytokine-induced systemic inflammatory response. AKI caused in the viral infection has been analyzed from the available epidemiological studies. The proportion of patients developing AKI is significantly higher when they develop severe disease. Continuous renal replacement therapy (CRRT) is the most used blood purification technique when needed. The impact of COVID-19 infection on chronic kidney disease (CKD) and renal transplant patients is also discussed
\end{abstract}

Manuscript submitted May 4, 2020, accepted May 19, 2020

Published online June 4, 2020

aDivision of Nephrology, Department of Internal Medicine, Adventist Medical Center, Hanford, CA 93230, USA

bPhoebe Putney Memorial Hospital, Medical College of Georgia, Albany, GA 31701, USA

'Department of Internal Medicine, University of Toledo and Promedica Toledo Hospital, Toledo, $\mathrm{OH} 43606$, USA

${ }^{\mathrm{d}}$ Division of Geriatric Medicine, Department of Family Medicine, University of Toledo, OH 43614, USA

eDepartment of Psychiatry, Great Plains Health, North Platte, NE 69101, USA fWise Health System, Decatur, TX 76234, USA

gDepartment of Medicine, Interfaith Medical Center, Brooklyn, NY 11213, USA hDivision of Rheumatology, Department of Internal Medicine, Adventist Medical Center, Hanford, CA 93230, USA

iDivision of Medical Oncology, Department of Internal Medicine, Ashland Bellefonte Cancer Center, Ashland, KY 41169, USA

${ }^{\mathrm{j} C o r r e s p o n d i n g ~ A u t h o r: ~ S r e e d h a r ~ A d a p a, ~ D i v i s i o n ~ o f ~ N e p h r o l o g y, ~ D e p a r t m e n t ~}$ of Internal Medicine, Adventist Medical Center, Hanford, CA 93230, USA. Email: sreedharadapa@gmail.com

doi: https://doi.org/10.14740/jocmr4200 in the manuscript. No vaccine has been developed against the 2019$\mathrm{nCoV}$ virus to date. The critical aspect of management is supportive care. Several investigative drugs have been studied, drugs approved for other indications have been used, and several clinical trials are underway across the globe. Recently remdesivir has received emergency use authorization by the Food and Drug Administration (FDA) in the USA for use in patients hospitalized with COVID-19. Prevention of the infection holds the key to management. The patients with underlying kidney problems and renal transplant patients are vulnerable to developing COVID-19 infection.

Keywords: COVID-19; Acute kidney injury; Chronic kidney disease; Renal transplant

\section{Introduction}

A series of pneumonia cases from unexplained etiology in Wuhan, China, since December 2019 has created the public health concern and resulted in the identification of the viral cause. The initial reports of this viral infection were traced to the Huanan seafood market, Wuhan, China, and the etiological agent was identified as coronavirus [1]. The World Health Organization (WHO) has named the virus as 2019 novel coronavirus (2019-nCoV) on January 12, 2020 and has declared as public health emergency globally on January 30, 2020 [2]. The disease caused by the virus was named as coronavirus disease 2019 (COVID-19) on February 11, 2020 [3]. The International Committee on Taxonomy of Viruses designated the etiological coronavirus as severe acute respiratory syndrome coronavirus 2 (SARS-CoV-2) [4]. The number of cases has been multiplying at an alarming rate globally, resulting in mortality, and WHO has declared COVID-19 as pandemic on March 11, 2020 [5]. On May 2, 2020, there were 3.4 million confirmed cases of COVID-19 infection cases globally, and the number of reported deaths were 244,213 [6].

After SARS and Middle Eastern respiratory syndrome (MERS), COVID-19 is the third known zoonotic CoV infection, and all belong to the beta-CoV cluster [1]. There was no determination on the animal species carrying the nCoV. The preliminary studies showed that the $\mathrm{nCoV}$ is closely related to 
the coronavirus isolated from bats, postulating the theory of possible transmission from bats to humans [1]. The genome homology sequence of SARS-nCoV and SARS-CoV was $79.5 \%$, and SARS-nCoV had high homology with bat CoV [7]. All the studies suggest the evidence strongly that the SARS$\mathrm{nCoV}$ might have originated from the bats, the intermediate host in transmission to humans yet to be ascertained. Ji et al have found that snakes are the wildlife repositories for the SARS-nCoV [8].

Various studies have reported the epidemiological data on SARS-nCoV. Most of the studies have shown that elderly patients are affected, higher male preponderance, and mortality is higher in the elderly with severe comorbid conditions [9]. The case fatality rate was estimated at $14-15 \%$, depending on the case series described $[9,10]$. The median incubation period is 14 days, and the median time from the first symptom to death is less in the elderly [1]. Mode of transmission is through close contact with people who has an infection through respiratory droplets when the infected person coughs or sneezes. Another possible mechanism is through infected surfaces and objects.

\section{COVID-19 Infection and Incidence of Renal Failure}

Epidemiological data revealed the severe illness rate of COVID-19 infection is as high as $25 \%$, and even though the lungs are the main organs affected, the kidney is also one of the main organs affected in severe illness [11]. Acute kidney injury (AKI) was seen in $5-15 \%$ of the cases infected with SARS$\mathrm{CoV}$ and MERS-CoV, and had a higher mortality rate of 60 $90 \%$ as per the literature [12]. COVID-19 infection causes AKI and is an independent risk factor for mortality [13, 14]. It also impacts patients with chronic kidney disease (CKD), patients on chronic replacement therapies, and patients with a kidney transplant. Mohamed et al [15] evaluated the contributing factors for developing AKI in 161 intensive care unit (ICU) patients. In this study incidence of AKI was $28 \%$. Approximately $35 \%$ of the patients who developed AKI had a history of CKD stages 3 - 5. Twenty-eight percent of the patients with CKD stages 3 - 5 had no AKI [15].

\section{Pathogenesis}

After the SARS-CoV2 spike (S) protein attaches to angiotensin-converting enzyme 2 (ACE2) receptors, the S protein is cleaved and activated by transmembrane serine proteases family (TMPRSS), which allows the virus to release fusion peptide that aids in the membrane fusion [16].

\section{Mechanism}

AKI in COVID-19 infection could be from the synergistic effect of virus-induced direct cytotropic effect and cytokineinduced systemic inflammatory response. AKI is more pronounced in patients with severe disease, acute respiratory dis- tress syndrome (ARDS), and those needing ICU admission. Other possible mechanisms of AKI could be from acute tubular necrosis (ATN) due to multiorgan failure and shock, and possible prerenal etiology from volume depletion secondary to decreased oral intake and high fever. Drug toxicity, hemodynamic insult, and contrast exposure can also play a role. The workup for AKI in COVID-19 infection should be similar to the other causes of AKI. Mohamed et al [15] discussed different etiologies of AKI in their study which include ischemic acute tubular injury, toxic acute tubular injury or combination of both, acute interstitial nephritis, de novo glomerular disease, pre-renal azotemia, and unspecified reasons. The contributing factors to different etiologies include hypotension, shock, rapid atrial fibrillation, prolonged volume depletion, rhabdomyolysis, toxic agents such as vancomycin, and iodinated contrast, overt proteinuria [15].

\section{Direct viral damage}

Prior studies have shown that positive SARS-CoV ribonucleic acid (RNA) polymerase gene fragments were detected by immunohistochemistry and in situ hybridization in kidney specimens of autopsy patients died from SARS [17]. MERS-CoV infections also have been shown to cause kidney epithelial cell damage by apoptosis mediated through receptors [18]. These studies suggest that the injury from coronavirus is a direct cytotoxic effect on kidney epithelial cells. Diao et al analyzed the kidney tissue in six patients on autopsy; immunohistochemistry demonstrated the presence of SARS-CoV-2 nucleocapsid (NP) protein in the kidney tubule, possibly related to potential direct tubular injury from the virus [19]. The SARS-CoV-2 RNA was detected in the urine of the patients by quantitative real-time polymerase chain reaction (qRT-PCR) [20].

\section{Role of $A C E 2$ receptors}

The normal human lung has type 1 and type 2 alveolar epithelial cells, which express ACE2 and is expressed in $83 \%$ of type 2 cells. Men have a higher expression rate of ACE2 levels compared to women, and similarly, Asians express higher ACE2 levels compared to white and African American populations [1]. The analysis by $\mathrm{Xu}$ et al found that ACE2 receptor expression in the kidney is no less than the lung on comparative analysis [16]. Podocytes and proximal convoluted tubule cells significantly co-express ACE2 and TMPRSS genes that are potential targets for SARS-CoV-2. The kidney tissue expresses the $A C E 2$ gene higher than that of lung tissue. The binding affinity of SARS-CoV-2 to ACE2 receptors is 10 - 20 times higher than SARS-CoV as described by Wrapp and colleagues [21].

\section{Immune-mediated damage}

The pro-inflammatory cytokine levels were elevated in COVID-19 infection and probable activation of T-cell response [9]. 
Table 1. Diagnosis of AKI Was Based on the KDIGO Classification

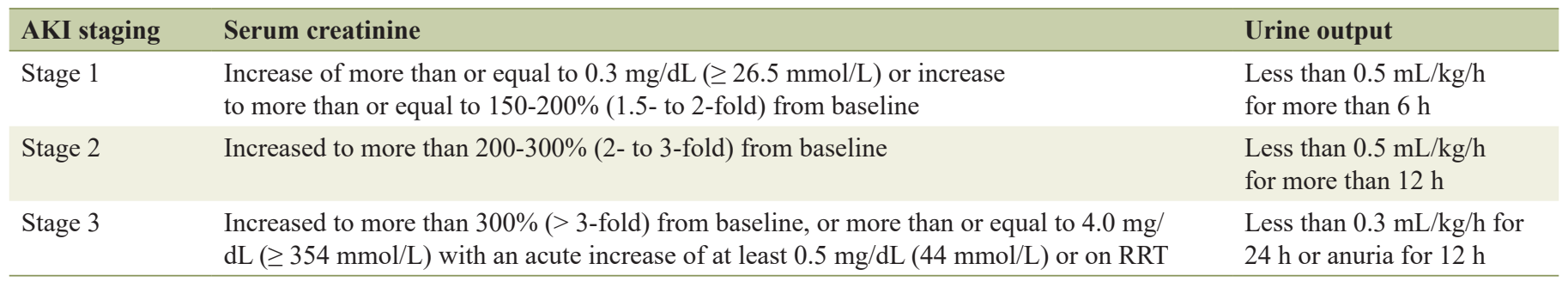

AKI: acute kidney injury; KDIGO: Kidney Disease Improving Global Outcomes; RRT: renal replacement therapy.

The possible occurrence of cytokine storm in severe cases is evidenced by the significantly higher cytokine levels. In the cytokine storm, the immune system damages healthy tissues rather than SARS-CoV-2 [16]. On the autopsies of six kidney tissues, the light microscopy revealed cluster of differentiation 68 (CD68) $^{+}$macrophage infiltration of the tubulointerstitium and severe ATN. The tubules showed complement 5b-9 deposition in all six cases, but deposition in glomeruli and capillaries were seldom seen. Some $\mathrm{CD}^{+} \mathrm{T}$ lymphocyte cells and $\mathrm{CD}^{+} 6^{+}$(natural killer) cells were seen in kidney tissue [19].

\section{COVID-19 Infection and AKI}

Recently published studies in China showed that the incidence of AKI in patients infected with COVID-19 is around 3-15\% $[22,23]$. In patients with severe COVID-19 infection in ICU, the rates of AKI increased significantly to $14.5-50 \%[23,24]$. The variation in reporting the incidence of AKI depends on the number of patients, severity of infection, and reporting of AKI across the studies. The diagnosis of AKI based on kidney disease improving global outcomes (KDIGO) classification was summarized in Table 1 [25].

\section{Cohort studies}

Cheng and colleagues analyzed a large cohort of 710 patients for kidney disease with confirmed COVID-19 admitted to Tongji Hospital from January 28, 2020 to February 11, 2020 [22]. All the patients were above 18 years of age. Patients who have a history of renal transplant and on maintenance dialysis were excluded from the study. The baseline serum creatinine was elevated in 110 patients on admission.

The patients in the elevated serum creatinine group were elderly, had a higher percentage of males, and the days from onset of illness to hospital admission were shorter. These patients also had severe disease and co-morbidities compared to those with normal baseline serum creatinine. These patients had more pronounced AKI and higher rates of mechanical ventilation, and the hospital mortality rate was higher.

Patients with elevated serum creatinine also had leukocytosis, lymphopenia, thrombocytopenia, prolonged activated partial thromboplastin time, and higher D-dimer levels. The patients with elevated baseline serum creatinine had a higher percentage of increased procalcitonin, aspartate aminotrans- ferase, and lactic dehydrogenase levels.

Most of the AKI occurred within 7 days, but was much quicker and severe in elevated baseline serum creatinine group. Patients with normal baseline creatinine had later onset of AKI and recovered quickly. There was elevated blood urea nitrogen (BUN), creatinine, high-grade proteinuria, and hematuria in elevated baseline serum creatinine patients along with significantly lower estimated glomerular filtration rate (eGFR). There was a significant gap between peak and baseline creatinine in the elevated serum creatinine group. Patients with elevated serum creatinine from baseline had worse outcomes compared to patients with normal baseline serum creatinine.

The study by Li et al [14] analyzed 59 cases of inpatients from multiple hospitals around Wuhan from January 21, 2020 to February 7, 2020 with COVID-19 disease, and 28 patients were diagnosed with severe cases according to the Chinese Nation Health guidance. Three of the patients died in the severe cases [14]. Proteinuria was present in $63 \%$ of the patients, and many of them had detected protein on the first day of admission, suggesting the presence of prior renal impairment. BUN was elevated in $27 \%$ of the patients, and an also higher level of BUN was present in two patients who died. Serum creatinine was elevated in $19 \%$ of the patients, and the patients who died had extremely high levels. The density was reduced on the computed tomography (CT) images of the kidney suggestive of edema and inflammation.

In a study by Wang et al [26], the patients needing ICU admission had higher BUN and creatinine levels at hospital admission compared to non-ICU admission patients and overall patients in general. The BUN and creatinine levels continued to get worse in non-survivors until the death [26].

We summarized the finding from multiple studies including patient characteristics, co-morbidities, incidence of AKI in general as well as ICU/severely ill patients, number of patients requiring continuous renal replacement therapy (CRRT), extracorporeal membrane oxygenation (ECMO) and mortality in Table 2 [9-11, 13, 19, 22-24, 26-32].

\section{Renal replacement therapies (RRTs) in hospital}

In the patients who developed AKI from COVID-19 infection, around $1.5-9 \%$ of them required RRT in the form of CRRT $[26,28]$. The proportion of patients needing RRT increased to $5.2-25 \%$ with severe infection $[11,27]$. As per the international experts, in severe COVID-19 cases with hypoxemic respira- 


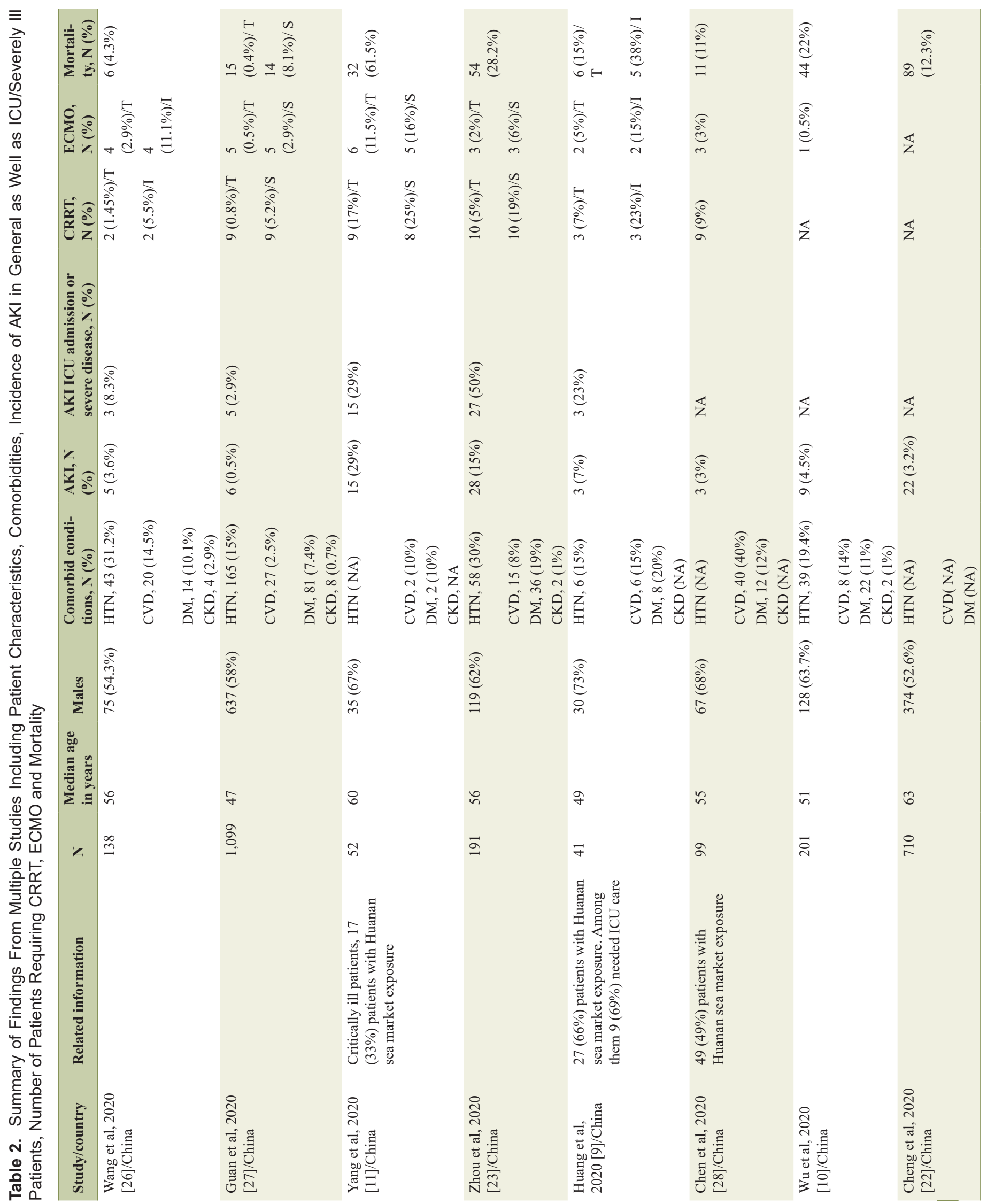




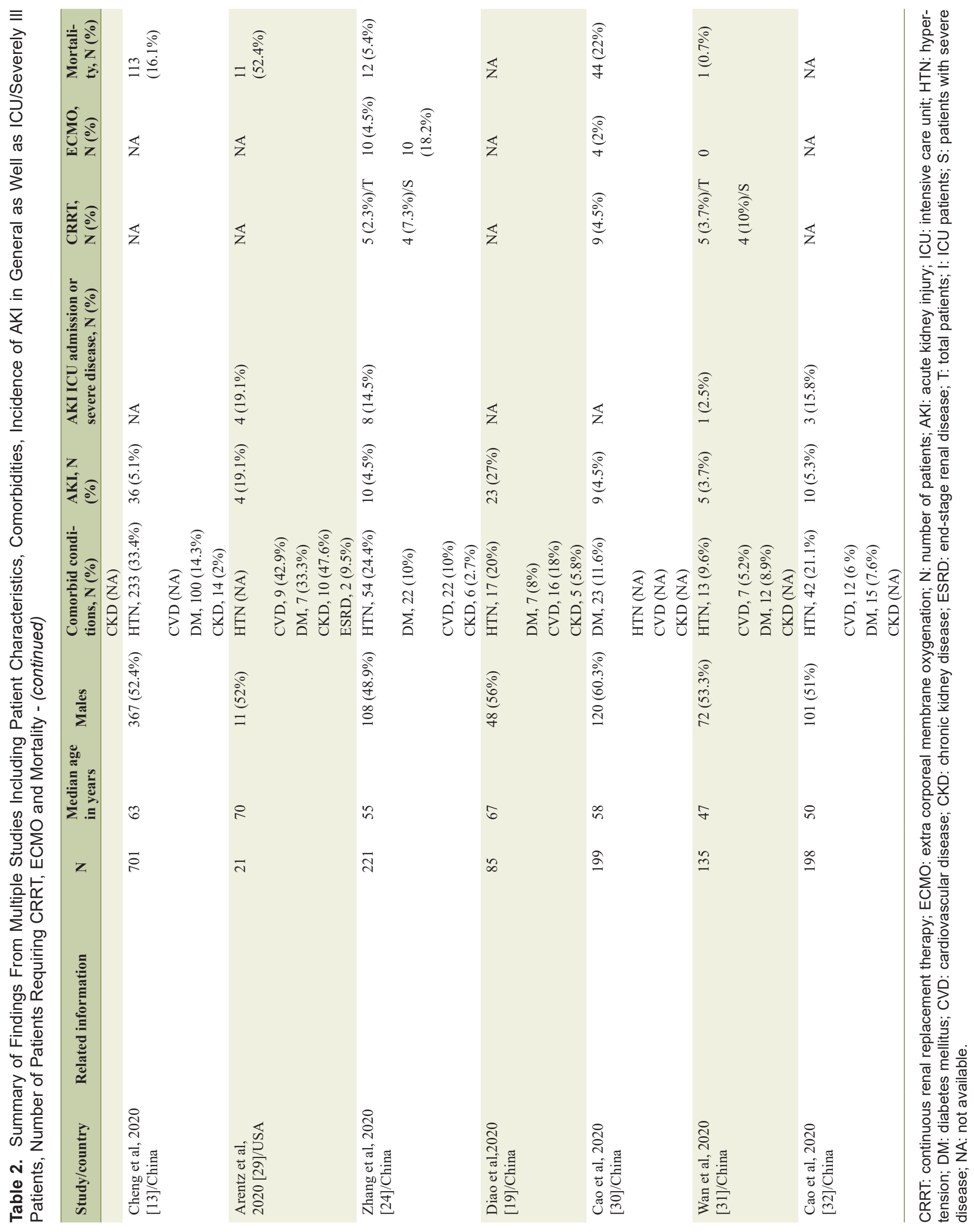


tory failure along with renal or liver dysfunction, ECMO with or without CRRT, can be used. CRRT is the most used blood purification modality in clinical practice, but for severe COVID-19 patients with sepsis and ARDS, hemoperfusion/plasma adsorption treatment can also be selected to clear more inflammatory mediators [33].

Recommendations on the care of hospitalized patients with COVID-19 and kidney failure needing RRT from the American Society of Nephrology (ASN) was summarized below [34].

\section{General measures}

All the health care personnel should follow the Centers for Disease Control and Prevention (CDC)-recommended personal protective equipment (PPE) and safety guidelines during their interactions with the patients. All the health care personnel should work as a team to limit the exposure to the infection. Indications for RRT are similar to other patients with AKI, and providers with significant expertise should place dialysis catheters.

\section{Patients in ICU}

The patients should be cohorted in dedicated ICUs, if possible. The preferred dialysis modality is CRRT or slow, low-efficiency dialysis (SLED) if available. Intermittent hemodialysis (IHD) can also be performed if CRRT and SLED are not available. CRRT is preferred over IHD in the setting of isolation, as IHD needs one-on-one nursing support. The patients needing CRRT exceeds the machines available, prolonged intermittent treatments (e.g., $10 \mathrm{~h}$ instead of continuous) with higher flow rates (e.g., $40-50 \mathrm{~mL} / \mathrm{kg} / \mathrm{h}$ ) and after terminal cleaning, can be used for another patient.

\section{Patients in general hospital floors}

The patients should be cohorted on the dedicated floor, if possible. Patients with active or suspected COVID-19 needing dialysis treatment should not be transported to a central acute dialysis unit. IHD should be provided with one-on-one nursing care if the patient is in negative pressure room. If all the COVID-19 patients are isolated on one floor, one dialysis nurse may be able to monitor two or three patients during IHD if video/ electronic monitoring is available. The nurse enters the room if the patient needs anything or to troubleshoot the machine. The peritoneal dialysis can be continued as automated peritoneal dialysis (APD) to limit the exposure of medical staff.

\section{Care and disinfection of RRT equipment}

The dialysis equipment should be cleaned with the disinfectant before removing it from the room. Hospital infection control and policy directs the proper disposable of RRT machine equipment. As per the hospital protocol, CRRT filters need to be changed every $72 \mathrm{~h}$ or longer.

Continuous venovenous hemofiltration, also known as $\mathrm{CVVH}$ or continuous venovenous hemodiafiltration (CVVHDF) are the preferred techniques. In the USA, continuous venovenous hemodialysis (CVVHD) is the most common modality used. Volume overloaded patients can be treated using slow continuous ultra-filtration (SCUF) to keep fluid balance net negative [35].

Temporary dialysis catheters are recommended for the vascular access and the providers with significant expertise should place dialysis catheters. It is recommended to use double-layered protection for the personnel performing the procedure. Filters with high ultrafiltration coefficient and high molecular weight cut-off membrane with $8-10 \mathrm{~nm}$ membrane pore size are suggested [36]. This would help in clearing high molecular weight inflammatory mediators. However, albumin would need to be replaced as it can be removed concurrently. It is essential to assess the patient's bleeding risk before choosing anti-coagulation. Patients with severe COVID-19 infection having high bleeding risk, it is recommended to avoid anticoagulation. In normal or hypercoagulable states, one can use heparin or citrate-based anticoagulation depending on hospital protocols. When using CRRT in combination with ECMO, anticoagulation is not needed as ECMO utilizes systemic heparinization [35].

It is essential to pay a very close attention to hemodynamics and volume status of patients with COVID-19 infection by using arterial and central venous pressure (CVP) monitors. Patients should have frequent renal panels checks every 4 - 6 $\mathrm{h}$ and monitor strict input and output to maintain a net negative fluid balance in ARDS patients. In patients without ARDS, the volume status should be assessed to maintain in euvolemic state.

The patients should be monitored for recovery of kidney function on daily basis, and the RRT should be discontinued once the patient is non-oliguric, euvolemic and improved ventilation requirements. This should also be supported by improved laboratory findings without alarming electrolyte or acid-base imbalance.

\section{Strategies to limit AKI}

AKI was associated with increased in-hospital mortality, so the patient should be meticulously managed by providing hemodynamic support, avoiding non-steroidal anti-inflammatory drugs (NSAIDs), nephrotoxins, and contrast. Early institution of CRRT will improve the outcomes. Proteinuria and hematuria were also associated with in-hospital mortality, so close attention should be paid to urine analysis, both in-patient and out-patient settings.

\section{AKI and mortality with COVID-19 infection}

The experience from various published studies on patients infected with COVID-19 infection showed that AKI is associ- 
ated with mortality. Shi et al analyzed 101 patients who died from COVID-19 infection showed that 23\% had AKI and $11 \%$ of patient had underlying CKD. BUN and myoglogin levels were higher in patients who died within 3 days and median time from hospitalization to death is 4 days [37]. Cheng in his analysis showed that elevated baseline serum creatinine, elevated BUN, peak serum creatinine $>1.5$, proteinuria, hematuria, AKI stages 2 and 3 are all associated with mortality after adjusting for confounding factors [22].

\section{COVID-19 Infection and CKD}

The impact of COVID-19 infection on CKD has not been reported. The incidence of known CKD patients varied from $0.7-$ $47.6 \%$, depending upon the series described [27, 29]. There was increased mortality in patients described in one study [29]. The incidence of AKI was higher in patients with established CKD [22].

The studies analyzed the kidney function in patients admitted with confirmed COVID-19 infection revealed the incidence of proteinuria and hematuria $[14,22]$. A significant number of patients had proteinuria on the day of admission, which could be related to the cytotropic effects of the virus on the podocytes. Proteinuria may result from direct podocyte injury from an expression of ACE2 [16].

The patients who recovered from the COVID-19 infection with proteinuria and hematuria need to be followed closely for the resolution. The patients who have sustained AKI during the infection need to be monitored for CKD.

\section{Renal Transplant and COVID Infection}

The ongoing outbreak of COVID-19 has amassed great concern worldwide, but its influence on transplant recipients is unknown. Previously experienced coronavirus outbreaks have taught us that transplant recipients can be easily affected by these infections $[38,39]$. With this being said, very little is known about the risk of the donor to receive transmission of the disease. Several factors concerning donor exposure like infectivity, incubation period, etc., can play an important role. Although there are no strict guidelines about donor restrictions, it would be advisable for potential donors to hold off on organ donation if suffering from a respiratory illness. The American Red Cross and European Centre for Disease Prevention and Control (ECDC) recommend 28-day and 21-day period delay respectively for potential organ donors with a history of recent travel to high-risk areas or contact with a person with suspected or proven COIVD-19 [40,41]. The ECDC recommends a 28-day delay after recovery from a confirmed infection. Michaels and colleagues recently well described possible risks associated with transplants in COVID-19-positive recipients [42]. Andrea et al from Italy described in their experience that in their institution, they have been limiting liver transplantation to the most urgent cases for the residents in the epidemic area [43].

A more liberal allocation policy is used for patients out- side epidemic areas. However, all recipients are screened to avoid transplantation in SARS-CoV-2-positive subjects. The internal board consistently reviews and updates the transplant list every 2 weeks according to the new insights on COVID-19. They continuously train their healthcare providers and screen them with nasopharyngeal swabs at frequent intervals. Providing timely information to patients and their caregivers to raise awareness and at the same time to avoid panic and confusion is important [43].

As per the review of the literature to the best of our knowledge, only one case of COVID-19 infection has been described in kidney transplant recipients of 12 years [44]. The patient was successfully managed initially with the cessation of all immunosuppression. He was given intravenous stress dose steroids to prevent adrenal insufficiency. Once the patient improved clinically, immunosuppression was resumed gradually. The overall clinical characteristics, including the symptoms, radiological findings on lung imaging, and laboratory findings, were similar to those of other non-transplanted adult patients with COVID-19 pneumonia. This being the only case reported so far, it is difficult to say that it is not possible to see atypical findings or more severe presentations of COVID-19 disease in transplant recipients.

Healthcare providers should be aware of that the experimental drugs like remdesivir and lopinavir/ritonavir could cause drug-drug interactions with calcineurin inhibitors [42]. It is therefore recommended to use these drugs with extreme caution. Regarding the treatment of kidney transplant recipients with COVID-19 infection, there is not much information available to help guide therapy and immunosuppression regimens. For now, it would be recommended to use due diligence and tailor management and treatment options based on the patient's clinical status, duration of transplant, and severity of illness. As the pandemic progresses, we now expect that we will have more information available for guidance.

\section{Treatment}

No vaccine has been developed against the SARS-CoV-2 virus to date. The critical aspect of management is supportive care. Multiple treatments such as empiric antibiotics, antiviral therapy, and systemic corticosteroids have been used worldwide. Several investigative drugs have been studied, drugs approved for other indications have been used, and several clinical trials are underway all across the globe.

One of the most promising of these therapies is remdesivir. It inhibits viral replication by premature termination of RNA transcription, and has shown activity against beta coronaviruses and in vitro activity against SARS-CoV-2 [45]. It has received emergency use authorization by the Food and Drug Administration (FDA) in the USA for use in patients hospitalized with COVID-19. It is recommended for 5 days in patients who are not on mechanical ventilation or ECMO, and recommended for 10 days in patients who are on mechanical ventilation or ECMO [46]. It is not recommended in patients with lower GFR (GFR $<30 \mathrm{~mL} / \mathrm{min})$ [47].

Others drug that has gained more attention was chloro- 
quine, hydroxychloroquine either alone or in combination with azithromycin. Chloroquine and hydroxychloroquine both have in vitro activity against SARS-CoV-2 [45]. They are both used in several countries for treating COVID-19 infection based on anecdotal data and in-vitro studies. There was a news report from China, where chloroquine increased viral clearance, decreased disease progression, and improved radiological findings $[47,48]$. There was another clinical trial reported from France, which is a non-randomized open-label trial of 36 patients compared to hydroxychloroquine or a combination of hydroxychloroquine and azithromycin versus standard of care in the control group. The combination of hydroxychloroquine and azithromycin in six patients resulted in superior viral clearance compared with hydroxychloroquine monotherapy [49]. There was a recent study among 368 veterans, which found no benefit of hydroxychloroquine but associated with more risks due to its side effect profile [50]. These medications should be used with caution in patients with renal and hepatic impairment, and they can cause QT prolongation in the electrocardiogram.

Hydroxychloroquine use for pre and post-exposure prophylaxis and treatment for SARS-CoV-2 infection is currently under investigation. Several clinical trials are planned in the USA to test the usage of hydroxychloroquine for treatment and prophylaxis for COVID-19. Clinicians are using different doses of hydroxychloroquine for prophylaxis and treatment.

A recent clinical trial in China did not show the efficacy of lopinavir-ritonavir for the treatment of pneumonia for COVID-19 patients, but this trial was underpowered [30]. WHO launched a global mega trial called SOLIDARITY of four most promising coronavirus treatments (remdesivir; chloroquine and hydroxychloroquine; ritonavir/lopinavir; ritonavir/ lopinavir and interferon beta) [51].

When antiretroviral medications for coronavirus treatment are used like lopinavir and ritonavir, dose adjustments to medications during CRRT are not needed, as they are $90 \%$ protein bound. Particular attention should be paid to the dosing of the drugs.

Other drugs are interferon beta, interferon alpha, mefloquine, favipiravir, darunavir, ribavirin, umifenovir, tocilizum$\mathrm{ab}$, and type II transmembrane serine protease (TMSPSS2) inhibitors. Support to therapies using vitamin A, B, C, D, E, thymosin alpha 1, thymopentin, Selenium, zinc and pyrithione combination have been used for treatment against SARSCOV-2 infection $[47,52]$.

\section{Conclusions}

COVID-19 infection is spreading rapidly and causing mortality daily worldwide. Unfortunately, knowledge about the novel virus is limited, and it causes a significant clinical threat to the general population and healthcare workers. Several countries have imposed strict regulations on the public to limit the spread of the virus. Many government agencies like CDC, FDA, and hospitals, public health systems are working every day in the USA to contain the spread of infection.

Studies have shown that there is the involvement of kidneys with COVID-19 infection and can be associated with high mortality. Health care providers should recognize this aspect early, and appropriate management should be instituted as soon as possible. The patients with underlying kidney problems and renal transplant patients are vulnerable to developing COVID-19 infection.

There is limited understanding of the pathogenesis of the disease, virulence of the virus at this moment, so prevention is the critical aspect in the management. Several vaccines and promising treatments are undergoing clinical trials with the hope of finding a cure for this global crisis soon.

\section{Acknowledgments}

None to declare.

\section{Financial Disclosure}

None to declare.

\section{Conflict of Interest}

None to declare.

\section{Author Contributions}

All authors contributed equally. SA, AC, MB, GPM, NMK, $\mathrm{SRD}, \mathrm{VG}, \mathrm{SN}$, and VMK were involved in review, and preparation of the manuscript. SA and VMK were involved in the analysis of data, final review of the manuscript, preparation of tables as well as submission. All the authors reviewed the manuscript and agreed with the findings and interpretation.

\section{Data Availability}

The authors declare that data supporting the findings of this study are available within the article.

\section{Abbreviations}

WHO: World Health Organization; 2019-nCoV: 2019 novel coronavirus; COVID-19: coronavirus disease 2019; SARSCoV-2: severe acute respiratory syndrome coronavirus 2; MERS: Middle Eastern respiratory syndrome; AKI: acute kidney injury; CKD: chronic kidney disease; ACE2: angiotensinconverting enzyme 2; TMPRSS: transmembrane serine proteases family; RNA: ribonucleic acid; qRT-PCR: quantitative real-time polymerase chain reaction; ICU: intensive care unit; KDIGO: Kidney Disease Improving Global Outcomes; ARDS: acute respiratory distress syndrome; ATN: acute tubular necrosis; BUN: blood urea nitrogen; eGFR: estimated glomerular filtration rate; CT: computed tomography; CRRT: continuous renal replacement therapy; ECMO: extracorporeal membrane 
oxygenation; ASN: American Society of Nephrology; CDC: Centers for Disease Control and Prevention; PPE: personal protective equipment; SLED: slow low efficiency dialysis; IHD: intermittent hemodialysis; APD: automated peritoneal dialysis; CVVH: continuous venovenous hemofiltration; CVVHDF: continuous venovenous hemodiafiltration; CVVHD: continuous venovenous hemodialysis; SCUF: slow continuous ultra-filtration; CVP: central venous pressure; NSAIDS: nonsteroidal anti-inflammatory drugs; ECDC: European Centers for Disease Prevention and Control

\section{References}

1. Sun P, Lu X, Xu C, Sun W, Pan B. Understanding of COVID-19 based on current evidence. J Med Virol. 2020.

2. Statement on the second meeting of the International Health Regulations. 2005. Emergency Committee regarding the outbreak of novel coronavirus (2019-nCoV). https://www. who.int/news-room/detail/30-01-2020-statement-on-thesecond-meeting-of-the-international-health-regulations(2005)-emergency-committee-regarding-the-outbreakof-novel-coronavirus-(2019-ncov).

3. World Health Organization. Director-General's remarks at the media briefing on 2019-nCoV on 11 February 2020. https://www.who.int/dg/speeches/detail/who-directorgeneral-s-remarks-at-the-media-briefing-on-2019-ncovon-11-february-2020. Accessed on February 12, 2020.

4. Coronaviridae Study Group of the International Committee on Taxonomy of $\mathrm{V}$. The species Severe acute respiratory syndrome-related coronavirus: classifying 2019-nCoV and naming it SARS-CoV-2. Nat Microbiol. 2020;5(4):536544.

5. World Health Organization. Director-General's remarks at the media briefing on COVID-19 on 11 March 2020. https://www.who.int/dg/speeches/detail/who-directorgeneral-s-opening-remarks-at-the-media-briefing-oncovid-19---11-march-2020.

6. John Hopkins University in medicine coronavirus resource Center. Available at: https://coronavirus.jhu.edu/map.html. Accessed on May 3, 2020.

7. Zhou P, Yang XL, Wang XG, Hu B, Zhang L, Zhang W, Si HR, et al. A pneumonia outbreak associated with a new coronavirus of probable bat origin. Nature. 2020; 579(7798):270-273.

8. Ji W, Wang W, Zhao X, Zai J, Li X. Homologous recombination within the spike glycoprotein of the newly identified coronavirus may boost cross-species transmission from snake to human. J Med Virol. 2020.

9. Huang C, Wang Y, Li X, Ren L, Zhao J, Hu Y, Zhang L, et al. Clinical features of patients infected with 2019 novel coronavirus in Wuhan, China. Lancet. 2020; 395(10223):497-506.

10. Wu P, Hao X, Lau EHY, Wong JY, Leung KSM, Wu JT, Cowling BJ, et al. Real-time tentative assessment of the epidemiological characteristics of novel coronavirus infections in Wuhan, China, as at 22 January 2020. Euro Surveill. 2020;25(3).

11. Yang X, Yu Y, Xu J, Shu H, Xia J, Liu H, Wu Y, et al.
Clinical course and outcomes of critically ill patients with SARS-CoV-2 pneumonia in Wuhan, China: a single-centered, retrospective, observational study. Lancet Respir Med. 2020;8(5):475-481.

12. Naicker S, Yang CW, Hwang SJ, Liu BC, Chen JH, Jha V. The Novel Coronavirus 2019 epidemic and kidneys. Kidney Int. 2020;97(5):824-828.

13. Cheng Y, Luo R, Wang K, Zhang M, Wang Z, Dong L, $\mathrm{Li} \mathrm{J}$, et al. Kidney disease is associated with in-hospital death of patients with COVID-19. Kidney International. 2020.

14. Li Z, Wu M, Yao J, Guo J, Liao X, Song S, Li J, et al. Caution on Kidney Dysfunctions of COVID-19 Patients. 2020. Available at SSRN: https://ssrn.com/abstract=3559601.

15. Mohamed MMB, Lukitsch I, Torres-Ortiz AE, Walker JB, Varghese V, Hernandez-Arroyo CF, Alqudsi M, et al. Acute kidney injury associated with coronavirus disease 2019 in Urban New Orleans. Kidney 360. 2020;1(4).

16. Pan XW, Xu D, Zhang H, Zhou W, Wang LH, Cui XG. Identification of a potential mechanism of acute kidney injury during the COVID-19 outbreak: a study based on single-cell transcriptome analysis. Intensive Care Med. 2020.

17. Ding Y, He L, Zhang Q, Huang Z, Che X, Hou J, Wang $\mathrm{H}$, et al. Organ distribution of severe acute respiratory syndrome (SARS) associated coronavirus (SARS-CoV) in SARS patients: implications for pathogenesis and virus transmission pathways. J Pathol. 2004;203(2):622-630.

18. Yeung ML, Yao Y, Jia L, Chan JF, Chan KH, Cheung KF, Chen $\mathrm{H}$, et al. MERS coronavirus induces apoptosis in kidney and lung by upregulating Smad7 and FGF2. Nat Microbiol. 2016;1:16004.

19. Diao B, Feng Z, Wang C, Wang H, Liu L, Wang C, Wang $\mathrm{R}$, et al. Human kidney is a target for novel severe acute respiratory syndrome coronavirus 2 (SARS-CoV-2) Infection. medRxiv. 2020.

20. Peng L, Liu J, Xu W, Luo Q, Deng K, Lin B, Gao Z. 2019 Novel Coronavirus can be detected in urine, blood, anal swabs and oropharyngeal swabs samples. medRxiv. 2020.

21. Wrapp D, Wang N, Corbett KS, Goldsmith JA, Hsieh CL, Abiona O, Graham BS, et al. Cryo-EM structure of the 2019-nCoV spike in the prefusion conformation. Science. 2020;367(6483):1260-1263.

22. Cheng Y, Luo R, Wang K, Zhang M, Wang Z, Dong L, Li $\mathrm{J}$, et al. Kidney impairment is associated with in-hospital death of COVID-19 patients. medRxiv. 2020.

23. Zhou F, Yu T, Du R, Fan G, Liu Y, Liu Z, Xiang J, et al. Clinical course and risk factors for mortality of adult inpatients with COVID-19 in Wuhan, China: a retrospective cohort study. Lancet. 2020;395(10229):1054-1062.

24. Zhang G, Hu C, Luo L, Fang F, Chen Y, Li J, Peng Z, et al. Clinical features and short-term outcomes of 221 patients with COVID-19 in Wuhan, China. J Clin Virol. 2020;127:104364.

25. Kidney International Supplement. https://kdigo.org/wpcontent/uploads/2016/10/KDIGO-2012-AKI-GuidelineEnglish.pdf.

26. Wang D, Hu B, Hu C, Zhu F, Liu X, Zhang J, Wang B, 
et al. Clinical characteristics of 138 hospitalized patients with 2019 novel coronavirus-infected pneumonia in Wuhan, China. JAMA. 2020;323(11):1061-1069.

27. Guan WJ, Ni ZY, Hu Y, Liang WH, Ou CQ, He JX, Liu L, et al. Clinical Characteristics of Coronavirus Disease 2019 in China. N Engl J Med. 2020;382(18):1708-1720.

28. Chen N, Zhou M, Dong X, Qu J, Gong F, Han Y, Qiu Y, et al. Epidemiological and clinical characteristics of 99 cases of 2019 novel coronavirus pneumonia in Wuhan, China: a descriptive study. Lancet. 2020;395(10223):507-513.

29. Arentz M, Yim E, Klaff L, Lokhandwala S, Riedo FX, Chong M, Lee M. Characteristics and Outcomes of 21 Critically Ill Patients With COVID-19 in Washington State. JAMA. 2020.

30. Cao B, Wang Y, Wen D, Liu W, Wang J, Fan G, Ruan L, et al. A Trial of Lopinavir-Ritonavir in Adults Hospitalized with Severe Covid-19. N Engl J Med. 2020;382(19): 1787-1799.

31. Wan S, Xiang Y, Fang W, Zheng Y, Li B, Hu Y, Lang C, et al. Clinical features and treatment of COVID-19 patients in northeast Chongqing. J Med Virol. 2020.

32. Cao M, Zhang D, Wang Y, Lu Y, Zhu X, Li Y, Xue H, et al. Clinical Features of Patients Infected with the 2019 Novel Coronavirus (COVID-19) in Shanghai, China. medRxiv. 2020.

33. Ronco C, Navalesi P, Vincent JL. Coronavirus epidemic: preparing for extracorporeal organ support in intensive care. Lancet Respir Med. 2020;8(3):240-241.

34. American Society of Nephrology. https://www.asn-online.org/g/blast/files/AKI_COVID-19_Recommendations_Document_03.21.2020.pdf.

35. National Center for Professional Medical Quality Management and Control of Nephropathy, Blood Purification Therapy and Engineering Technology Branch of the Chinese Medical Advocacy Council, and the Blood Purification Therapeutic Committee of the Army. Expert opinion on CRRT application in the treatment of new coronavirus pneumonia [EB / OL]. 2020. www.cnrds.net/Static/OfficialDocumentDown.html.

36. Chen X. Standard operating procedures for blood purification. Beijing: People's Military Medical Publishing House. 2010.

37. Shi Q, Zhao K, Yu J, Feng J, Zhao K, Zhang X, Chen X, et al. Clinical characteristics of 101 non-surviving hospitalized patients with COVID-19: A single center, retrospective study. medRxiv. 2020.

38. Kumar D, Tellier R, Draker R, Levy G, Humar A. Severe Acute Respiratory Syndrome (SARS) in a liver transplant recipient and guidelines for donor SARS screening. Am J Transplant. 2003;3(8):977-981.

39. AlGhamdi M, Mushtaq F, Awn N, Shalhoub S. MERS $\mathrm{CoV}$ infection in two renal transplant recipients: case re- port. Am J Transplant. 2015;15(4):1101-1104.

40. Red Cross media statement on 2019 novel coronavirus. https://www.redcross.org/about-us/news-and-events/pressrelease/2020/red-cross-media-statement-on-2019-novelcoronavirus.html. Accessed on March 23, 2020.

41. Cluster of pneumonia cases caused by a novel coronavirus, Wuhan China. 2020. https://www.ecdc.europa.eu/sites/default/files/documents/Risk $\% 20$ assessment $\% 20 \% 20$ pneumonia\%20Wuhan\%20China\%2017\%20Jan\%202020.pdf. Accessed on March 23, 2020.

42. Michaels MG, La Hoz RM, Danziger-Isakov L, Blumberg EA, Kumar D, Green M, Pruett TL, et al. Coronavirus disease 2019: Implications of emerging infections for transplantation. Am J Transplant. 2020.

43. Gori A, Dondossola D, Antonelli B, Mangioni D, Alagna L, Reggiani P, Bandera A, et al. Coronavirus disease 2019 and transplantation: A view from the inside. Am J Transplant. 2020.

44. Zhu L, Xu X, Ma K, Yang J, Guan H, Chen S, Chen Z, et al. Successful recovery of COVID-19 pneumonia in a renal transplant recipient with long-term immunosuppression. Am J Transplant. 2020.

45. Wang M, Cao R, Zhang L, Yang X, Liu J, Xu M, Shi Z, et al. Remdesivir and chloroquine effectively inhibit the recently emerged novel coronavirus $(2019-\mathrm{nCoV})$ in vitro. Cell Res. 2020;30(3):269-271.

46. Food and Drug Administration. https://www.fda.gov/media/137564/download.

47. Sanders JM, Monogue ML, Jodlowski TZ, Cutrell JB. Pharmacologic Treatments for Coronavirus Disease 2019 (COVID-19): A Review. JAMA. 2020.

48. Gao J, Tian Z, Yang X. Breakthrough: Chloroquine phosphate has shown apparent efficacy in treatment of COVID-19 associated pneumonia in clinical studies. Biosci Trends. 2020;14(1):72-73.

49. Gautret P, Lagier JC, Parola P, Hoang VT, Meddeb L, Mailhe M, Doudier B, et al. Hydroxychloroquine and azithromycin as a treatment of COVID-19: results of an open-label non-randomized clinical trial. Int J Antimicrob Agents. 2020:105949.

50. Magagnoli J, Narendran S, Pereira F, Cummings T, Hardin JW, Sutton SS, Ambati J. Outcomes of hydroxychloroquine usage in United States veterans hospitalized with Covid-19. medRxiv. 2020.

51. WHO launches global megatrial of the four most promising coronavirus treatments. https://www.sciencemag.org/ news/2020/03/who-launches-global-megatrial-four-mostpromising-coronavirus-treatments.

52. Balla M, Merugu GP, Patel M, Koduri NM, Gayam V, Adapa S, Naramala S, et al. COVID-19, modern pandemic: a systematic review from front-line health care providers' perspective. J Clin Med Res. 2020;12(4):215-229. 